Article

\title{
Energy and Force Optimization of a Network of Novel Electromagnetic Soft Actuators
}

\author{
Nafiseh Ebrahimi and Amir Jafari * \\ Advanced Robotic Manipulators (ARM) Lab, Department of Mechanical Engineering, \\ University of Texas at San Antonio (UTSA), One Circle, San Antonio, TX 78249, USA; \\ nafiseh.ebrahimi@utsa.edu \\ * Correspondence: amir.jafari@utsa.edu; Tel.: +1-210-458-6544
}

Received: 21 April 2020; Accepted: 27 June 2020; Published: 10 July 2020

\begin{abstract}
This paper discusses how to optimally design polygonal profiles of Electromagnetic Soft Actuators (ESAs) to be used in a network to achieve maximum output force with minimum energy consumption. The soft actuators work based on operating principle of solenoids but are made of intrinsically soft materials. It was, previously, confirmed that by miniaturizing the size, the amount of output force decreases for a single ESA however, by the ratio of force to volume increases. Therefore, networking small sized ESAs, would increase the output force. Initially, ESAs were made with circular cross-section profiles. However, we prove here that the shape of the cross-section profile can affect the output force. A polygonal shape with fewer sides would result in higher output force for a single ESA. However, with a network of ESAs, another parameter, packing density, plays an important role in the output force. Our optimization results suggest that even though triangular cross-section profiles lead to the highest amount of force for a single ESA, the best choice would be hexagonal shapes when they are networked.
\end{abstract}

Keywords: design optimization; soft magnet; electromagnetic actuator

\section{Introduction}

Physical Human-Robot Interaction (pHRI), requires a technology to fabricate the robotic platform that is fundamentally different from industrial robots [1]. Examples are surgical robots, prosthetic devices and exoskeletons. These platforms are supposed to physically interact with the soft tissues of the human body, where exerting forces outside the tissues structural limitations would lead to medical hazards. Industrial robots are designed for fast and accurate position control applications where everything about the environment is known and predictable. In pHRI, however, we need to take into account the uncertainties regarding the force interactions [2]. An intrinsically soft robotic platform can provide a novel and safer option for biomedical applications [3,4].

The source of producing force into robotic platforms is actuators. Actuators have a critical role in the perceivable softness of the whole robotic platform. Recently, actuators with intrinsically soft bodies have been gaining interest among researchers and as a result different soft actuators have been proposed and fabricated. Pneumatic soft actuators [5-7] were actually the first types of soft actuators that were introduced. In these actuators, air pressure inside a stretchable tube, usually made of Polydimethylsiloxane (PDMS) [8], leads to inflate-deflate the tube and creates axial elongation-shrinkage. Therefore, this axial motion can create a desired amount of force. These actuators have high bandwidth, and output a relatively high amount of force and deflection. Looking at the whole picture, however, there has to be air pressure to be supplied by an air pump which is indeed bulky and rigid and also the actuator has to be tethered to the pump. This not only makes the entire robot's body "not soft" but also very huge if the source of power is taken into account. 
Another type of soft actuator is the shape memory alloy [9,10]. A shape-memory alloy (SMA) is deformed when it is cold; however, it regains its pre-deformed shape once it is heated. These actuators are indeed intrinsically soft and usually do not require a high amount of input power. However, they usually have very limited bandwidth, as it takes a long time for the alloy to cool down and retrieve its primary shape and compliance following electrical actuation.

Dielectric polymers [11-13] are another type of soft actuators. These elastomers are in fact smart materials that generate large amount of strain when voltage is being applied to them which is converted into mechanical work. High elastic energy density while being lightweight are advantages of these actuators. However, the most limiting disadvantage is the extensively high amount of voltage (in order of $20 \mathrm{kV}$ ) that these actuators need to be activated.

Another type of recently developed soft actuators works based on liquid-gas transition [14,15]. In these thermo-active soft actuators a liquid with low boiling point is encapsulated inside a soft and stretchable tube usually made of PDMS. Generating heat by introducing electric current through a resistive element leads to increasing the temperature. The increased temperature changes the phase from liquid to gas and consequently large amount of volume changes. This large change in the volume inflates the soft tube and so motion and force are generated. The disadvantage of this actuator is, however, its very limited bandwidth and therefore, very slow response.

According to the Lorentz law a conductive element carrying electrical current in a magnetic field will experience a force acting perpendicular to both the magnetic field and direction of electric current. Electromagnetic working principle of traditional rigid actuators $[16,17]$ has been implemented in another recent type of soft actuators. This type of soft actuators do not require high amount of input voltage as dielectric actuators do, and are fast in response and can develop considerably high amount of deflection. However, the challenge in these types of actuators is the relatively low amount of generated force. The reason is due to several factors: first of all, these actuators use a conductive liquid (usually Eutectic Gallium Indium or EGaIn) which have higher electrical resistance as compared to copper wires in traditional rigid actuators. Also, these actuators usually use flexible permanent magnets made by a mixture of PDMS and magnetic particles, which have lower magnetic strength as compared to rigid permanent magnets in traditional rigid actuators.

We have developed a novel Electromagnetic Soft Actuator (ESA) based on the working principle of solenoids with permanent magnets [18-20]. In our ESA, electromagnetic field is created by applying electric current through a soft conductive coil. The coil is made of PDMS micro-pipe with diameter of around $0.1 \mathrm{~mm}$ filled by EGaIN. In our design, two coils are antagonistically embedded inside a PDMS body with a springy connection in between as it is shown in Figure 1. Once electric current is being supplied the two coil can get magnetized based on the Lorentz law and attract each other. In order to intensify the electromagnetic field between the two coils, a flexible permanent magnet is placed inside the coils.

The permanent magnet is made by pouring a mixture of PDMS and magnetic particles into a 3D printed cylindrical-shaped mold, where the mixture in placed inside a strong external magnetic field while it is curing, to align the magnetic orientation of each particle. As a result, once the mixture is completely cured, the magnetic particles remain aligned even when the external magnetic field is abolished. The product is a cylindrical flexible material with magnetic properties.

This type of soft actuator is highly scalable where the scaling factor is determined by the available manufacturing technology. For example, the smallest PDMS micro-pipe available has a diameter around $0.1 \mathrm{~mm}$, whereas using high-end 3D printers, PDMS can be printed as a coil-shape with embedded helical micro-channel inside with diameter in the order of micrometers. However, due to extremely high price of such 3D printers, this manufacturing technology is very expensive and only justifiable in mass production. 


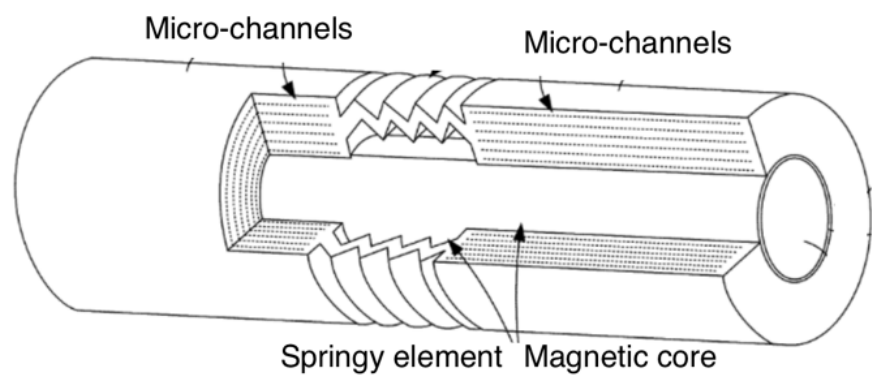

(a)

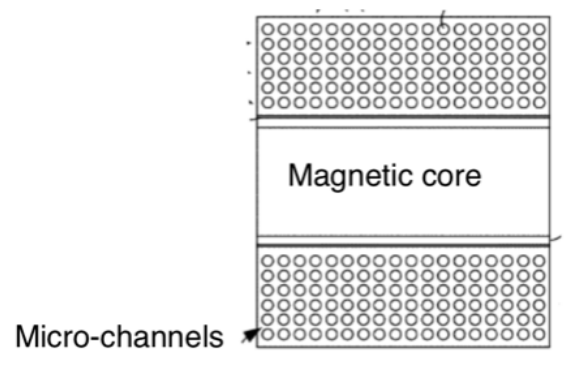

(b)

Figure 1. Design concept of an Electromagnetic Soft Actuator (ESA): (a) Schematic and (b) cross-section view.

Scaling factors for several actuation technologies, such as electromagnetic, piezoelectric and electrostatics have been studied by other researchers [21,22]. It has been shown that for electromagnetic actuators the force is proportional to square of the length. This means that by decreasing the size of the actuator, the generated force will be reduced. However, interestingly we found that by scaling down the ESA's size, its force to volume ratio increases. This was confirmed through experiments on different sizes of ESAs [20]. This behaviour was also analytically proven using the geometry of the actuator and electromagnetic equations based on the Lorentz Law [23]. The limitation on scaling down the size of ESA depends on available technology. Nano-Scribe 3D printer can create micro-scale size micro-channels but the cost of the 3D printer is about half of a million dollars.

This property of ESAs suggests that by reducing the size of ESAs and attaching them in series and parallel as a network, the output force can be enhanced compared to a single ESA with the same size of the networked ESAs. Due to their light weight, their fast response, and being able to be operated with voltage range around $10 \mathrm{~V}$ to $80 \mathrm{~V}$, with increased output force, ESAs can reproduce a soft actuation technology suitable for pHRI, especially for rehabilitation and support of patients with mobility impairments.

In order to examine the capability of a networked ESAs to be used as drive train for a rehabilitation or force augmentation device, we consider a case of an active elbow brace and use of optimization method to find the optimal structure of the network of ESAs.

\section{Materials and Methods}

\subsection{Design Parameters of Networked Electromagnetic Soft Actuators and Force Formulation}

In this section, we first formulate the force as a function of design parameters of a single and then double (antagonistic) coils. We then include the effect of magnetic core to calculate the resultant force. In the next step, the generated force of networked ESAs will be formulated as a function of its design parameters. 


\subsubsection{Electromagnetic Force of Two Antagonistic Coils}

As mentioned before, our proposed ESA consists of two electromagnetically inductive coils that contain a flexible permanent magnet core. The force generated by the coils depends on several parameters as following: number of turns, number of loops, length and diameter of the coils, electrical current, length and diameter of the flexible permanent magnet, and the type and ratio of mixture of materials being used.

To design the ESA, a theoretical analysis should be conducted on the electromagnetic force that is being produced by the two coils, taking into account that the two coils share a common flexible permanent magnetic core. For this purpose, we first calculate the magnetic field by the Biot-Savart Law [24] alongside the axis $z$ of a representative loop of the coil with unit vector of $\hat{k}$, considering an arbitrary point $\mathrm{P}$ through which a steady current $I$ is passing (Figure 2a).

By considering the magnetic field at point $\mathrm{P}$ due to the contribution of the current element and then integrating over the whole path of current on the circular loop. The first integral vanishes due to the fact that radial unit vectors all over the circle cancel out each other and result in zero. Therefore, the overall result would only be the axial part of the produced magnetic field as:

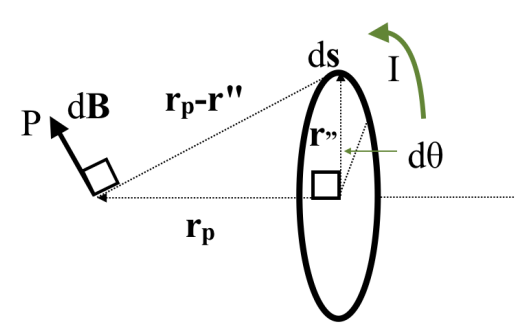

(a)

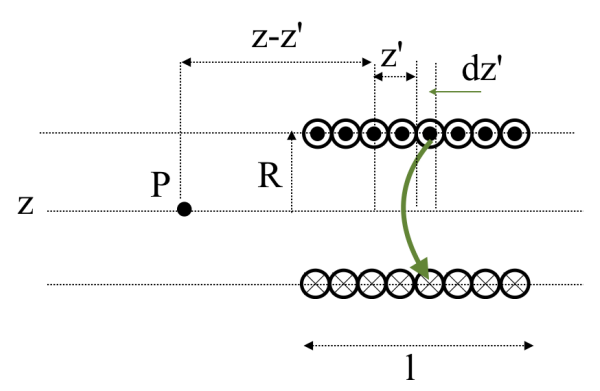

(b)

Figure 2. (a) Magnetic field at an arbitrary point $P$ resulted by a circular current carrying element and (b) magnetic field at an arbitrary point generated by a current carrying coil.

$$
\overrightarrow{\mathbf{B}}_{z}=\frac{\mu_{0} I R^{2}}{4 \pi\left(Z^{2}+r^{2}\right)^{3 / 2}} \int_{0}^{2 \pi} d \theta \hat{k}
$$

This is the magnetic field of a single loop acting on point $P$ along the axis $\hat{k}$. A solenoid consists of many loops and layers of loops. In order to calculate the magnetic field acting on an arbitrary point $P$ along the axis $\hat{k}$ in a solenoid, the consequent magnetic field of a single loop should be integrated over the entire length of the Solenoid.

Similarly by integrating $d \overrightarrow{\mathbf{B}_{z}}$ over the entire length of the solenoid, the total magnetic field at point $P$ can be found as:

$$
\overrightarrow{\mathbf{B}_{z}}=\frac{\mu_{0} n I}{2}\left[\frac{l / 2-z}{\left[(z-l / 2)^{2}+R^{2}\right]^{0.5}}+\frac{l / 2+z}{\left[(z+l / 2)^{2}+R^{2}\right]^{0.5}}\right]
$$


This is the produced magnetic field out of a coil at any given point $P$ along its central axis $\hat{k}$ by an axial distance $z$ from its central loop that is located along the axis $\hat{k}$ at the middle of solenoid's length.

In our ESA's design, two antagonistic coils are interacting with each other and therefore, contribution of both coils on a given point should be taken into account. By calculating magnetic field due to each coil (left and right) and then applying the superposition principle, we can find the total magnetic field at any given point $\mathrm{P}$ located on the common central axis of the two antagonistic coils.

To calculate each magnetic field, the distance of point $P$ from the central loop of its corresponding solenoid $\left(z_{1}\right.$ and $\left.z_{2}\right)$ must be calculated.

In our ESA design, as it is shown in Figure 3, the permanent magnet's North pole is located in the right coil while the South pole is located in the left coil. $d$ is the distance between each coil's mid point and the magnetic poles. Each pole has the equal distance to the corresponding solenoid's end as the magnetic core is placed precisely at the midpoint of the central line that is connecting the two coils' axes. Due to symmetrically antagonistic geometry of our design, only the applied force at one end surface of the magnetic core has to be calculated and then doubled to calculate the total force applied.



Figure 3. Schematic configuration of ESA composed of two solenoids and a shared magnetic core in the middle.

To find the magnetic field at any given point within this design, let us consider $z_{1}$ and $z_{2}$ to be $d$ and $d+h$, respectively. The total magnetic field due to left and right side coils can then be calculated as:

$$
\overrightarrow{\mathbf{B}}_{\text {ext }}=\frac{\mu_{0} n I}{2}\left[B_{1}+B_{2}+B_{3}+B_{4}\right]
$$

where $B_{1}=\frac{l / 2-d}{\left[(-d+l / 2)^{2}+R^{2}\right]^{0.5}}, B_{2}=\frac{l / 2+d}{\left[(-d-l / 2)^{2}+R^{2}\right]^{0.5}}, B_{3}=\frac{l / 2-(d+h)}{\left[((d+h)-l / 2)^{2}+R^{2}\right]^{0.5}}$ and $B_{4}=\frac{l / 2+(d+h)}{\left[((d+h)+l / 2)^{2}+R^{2}\right]^{0.5}}$.

The force that applied to either North or South Pole of the magnetic core and generated by magnetic fields of both the right and the left coils can be calculated as:

$$
F=\frac{n I B_{r}}{2} \pi \bar{r}^{2}\left[B_{1}+B_{2}+B_{3}+B_{4}\right]
$$

Therefore, the total force applied to the magnetic core both at the North and South poles would be $F_{\text {total }}=2 F$. The current in this equation can be determined by the input voltage $V_{i n}$ and equivalent resistance of the coil's circuit $R_{e q v}$ as $I=\frac{V_{i n}}{R_{e q v}}$. The equivalent resistance of the coil is a function of length $L_{w}$ and cross-section area $A_{w}$ of its conductive wire as well as specific resistivity of the conductive material $\rho_{w}$ as $R_{e q v}=\rho_{w} \frac{L_{w}}{A_{w}}$. For more information please refer to [20].

\subsubsection{Network of Electromagnetic Soft Actuators}

A network of ESAs consists of ESAs attached in series and parallel, similar to the arrangement of Actin and Myosin filaments in skeletal muscles. When ESAs are attached in series fashion, they compose a Fiber and when fibers are attached in parallel, they make a Fascicle. Output force of a 
fiber is equivalent to the output force of a single ESA while the total deflection of a fiber is summation of deflections of its ESAs.

The output force of a fascicle, however, is the summation of output force of its fibers, which, as mentioned before, is equivalent to the summation of output force of single ESAs attached in parallel. Therefore, to maximize the output force of a networked ESAs, in addition to maximizing the output force of a single ESA, the parallel arrangement of ESAs should be optimized. This parallel arrangement is a function of the cross-section profile of ESAs. Usually coils have circular cross-section profile, but the conductive wire can also be wrapped in different cross-section shapes such as triangular, square, etc., basically if $n$ represents number of sides of a shape, the cross-section profile can have $n$ sides where $n$ can change from 3 (i.e., triangle) to $\infty$ (i.e., circle).

The purpose of this subsection is to geometrically design a fascicle network of ESAs so that the magnetic field and force out of a certain occupied volume of actuator network can be maximized. In the previous subsection we formulated the magnetic field and output force of a single ESA mainly by taking into account the geometry of the coils embedded in it. Now, we intend to discuss the formulation of a network of soft actuators, which are positioned with their long axis parallel next to each other. Given the point that the coil is the heart of the proposed ESA, we need to study the coil's cross-section profiles. The idea is to eventually find the best cross-section profile for the axially packed network of solenoids shown in Figure 4 which yields to the maximum generated magnetic field and force out of a certain occupied volume of the network. Since, we intend to compare cross section profiles for each solenoid profile, we just need to determine the magnetic field and force of one loop coil.

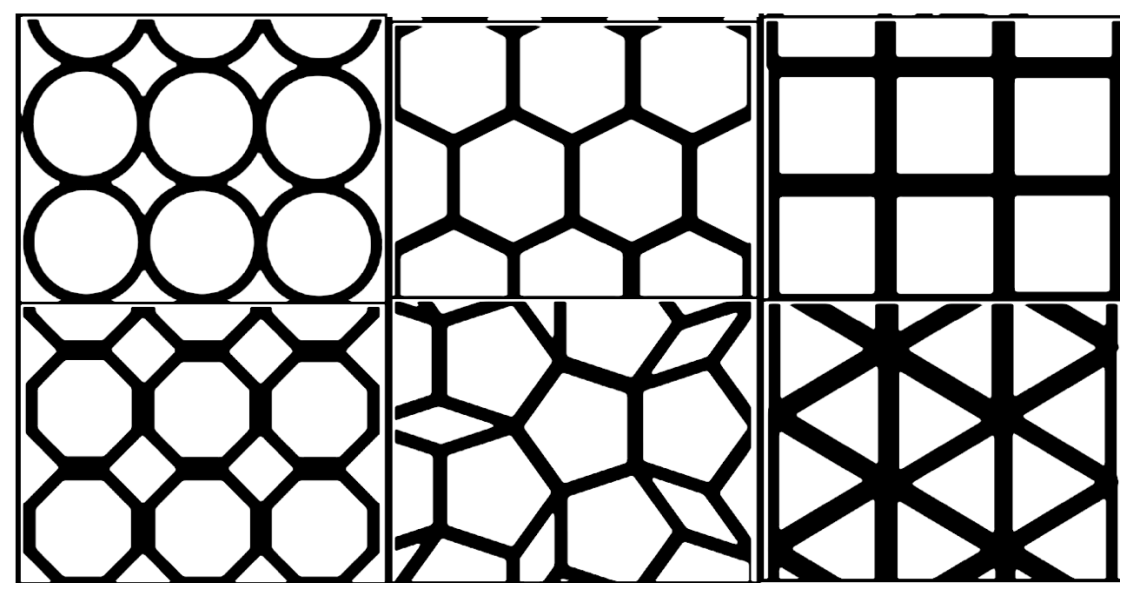

Figure 4. Triangular, square, pentagonal, hexagonal octagonal and, circular cross section shapes.

The polygons and circle shapes for the coil's section profiles will be studied here. For this purpose, similar to the approach was taken in previous subsection, the magnetic field of the considered profile needs to be determined using Biot-Savart Law. For all the polygonal profiles, firstly, we need to calculate the magnetic field generated by a finite straight line representing the side of the polygons at a desired point as shown in Figure 5a. 


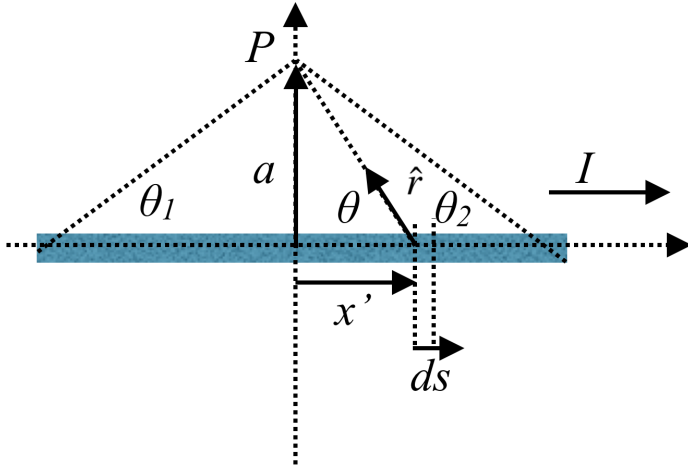

(a)

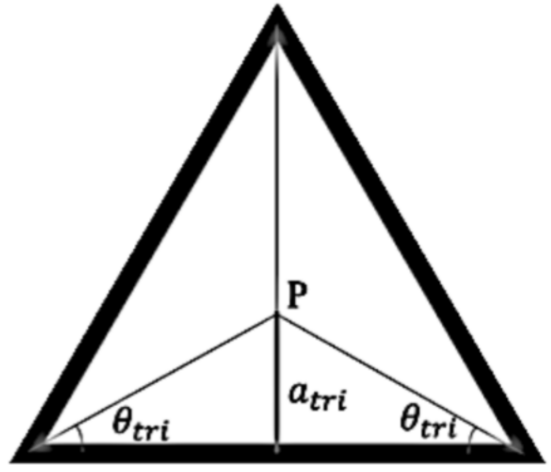

(b)

Figure 5. (a) Magnetic field of a piece of straight wire at an arbitrary point $\mathrm{P}$ and (b) triangular cross-section profile geometry.

The current carrying element in $x$ direction is $d \vec{s}=d x^{\prime} \hat{i}$. The goal is to find the magnetic field of the current carrying wire at point $\mathrm{P}$. The position vector describing $\mathrm{P}$ is $\vec{r}_{P}=a \hat{j}$. The relative position vector which points from the source point to the field point is $\vec{r}=\vec{r}_{P}-\vec{r}$. For point $P$ with the location of $(0, a)$, the relative position vector would be $\vec{r}=a \hat{j}-x^{\prime} \hat{i}$. The magnitude of this vector defines the distance between the current element and the point $P$ and is equal to $r=\left(a^{2}+(x \prime)^{2}\right)^{0.5}$. The corresponding unit vector is given by $\hat{r}=\frac{\vec{r}}{r}=\sin \theta \hat{j}-\cos \theta \hat{i}$.

According to the Biot-Savart Law the contribution to the magnetic field due to the current carrying element is:

$$
d \vec{B}=\frac{\mu_{0} I}{4 \pi} \frac{d \vec{s} \times \hat{r}}{r^{2}}
$$

Substituting $r=a \cos \theta$ and $d x=-a \csc ^{2} \theta d \theta$ in Equation (5), the differential contribution on the magnetic field is obtained as:

$$
d B=\frac{\mu_{0} I}{4 \pi a} \sin \theta d \theta
$$

Integrating this infinitesimal magnetic field over the range of angles from $-\theta_{1}$ to $\theta_{2}$, the total magnetic field can be calculated as:

$$
B=\frac{\mu_{0} I}{4 \pi a} \int_{-\theta_{1}}^{\theta_{2}} \sin \theta d \theta=\frac{\mu_{0} I}{4 \pi a} \cos \theta_{2}+\cos \theta_{1}
$$

In case of symmetrical arrangement of the conductive wire around perpendicular line passing through point $\mathrm{P}$ (i.e., $\theta_{1}=\theta_{2}=\theta$ ), the total magnetic field would be:

$$
B=\frac{\mu_{0} I}{2 \pi a} \cos \theta
$$

Now that we determined the magnetic field out of a finite wire carrying current, it is possible to calculate the magnetic field produced by polygon shaped current caring loops.

Next step, the magnetic field of triangular, square, pentagonal, hexagonal and, octagonal shape current carrying elements with the equal cross-section areas will be calculated. For each polygon shape, the magnetic field at the central point of the coil's section profile is obtainable by superposition of the magnetic field produced by each sides at the center of the profile. For instance, for a triangular profile, depicted in Figure 5b, the magnetic field at the central point of the section would be:

$$
B_{\text {tri }}=3 B_{\text {line }}=\frac{3 \mu_{0} I}{2 \pi a_{t r i}} \cos \theta_{\text {tri }}
$$


Therefore, in order to compare various cross-section geometries, we would equalize different sections in terms of cross-section area and find the correlated edge length of each polygon solenoid section. In other words, we assume that all polygon sections have same cross-section area and then the side's length for each one can be calculated.

Once, the magnetic field of all cross-section profiles has been calculated, the generated force derived from the interplay between coil and magnetic core can be obtained:

$$
F=B_{\text {coil }} \frac{B_{r}}{\mu_{0}} A_{\text {cross-section }}
$$

where $A_{\text {cross-section }}$ is the cross-section of the magnetic core inside the solenoid. For each coil profile geometry, the magnetic core has the same profile with the correlated coil. Since, we assumed earlier that all profiles have the same cross section area, the magnetic core cross-section $A_{\text {cross-section }}$ would be the same as well. Therefore, the produced force from the coil would be proportional to its magnetic field for each case, hence the ratio of generated forces would be the same as the ratio of magnetic fields.

\subsection{Case Study: an Active Elbow Brace}

As mentioned before, bio-inspired arrangement of ESAs can lead to an enhanced output force. If we can achieve the level of forces that are exerted physiologically at a human's extremity joints, by on-board batteries, this actuation technology can greatly impact rehabilitation or force augmentation applications for mobility impaired patients, as it is light weight, has high bandwidth, and it is powerful and portable.

To determine whether or not networked ESAs have this capability, we selected a human elbow joint, as a critically important joint in performing our daily activities. The elbow joint serves as a practical link between the forearm and the upper arm. It makes the forearm to function like a lever in carrying and lifting objects, stands the hand in space. It is also capable to provides accuracy in both closed and open kinetic chain work. Hence, even a mild destruction in this joint could remarkably deteriorate the functionality of the hand to perform perfectly. It could get more influential considering the fact that the other joints in the upper limb are not able to compensate for the elbow's failure.

We intend to first determine the performance requirement of an active elbow brace that is supposed to be worn by a patient and match the performance of a healthy elbow joint [25], in terms of available torque and angular deflection, i.e., flexion-extension. Range of elbow's flexion-extension in a healthy individual is between 0 to 140 degree, while the maximum torque, that the elbow can handle at 90 degree (flexed) is about $60 \mathrm{Nm}$. The other performance parameters of the active elbow brace that are summarized in Table 1, have been calculated based on available passive elbow braces in the market.

Table 1. Characteristics of an Active Elbow Brace.

\begin{tabular}{ccc}
\hline Characteristics & Value & Unit \\
\hline Range of Flexion-Extension & $0-140$ & Degree \\
Torque & $40-60$ & $\mathrm{Nm}$ \\
Weight & $150-300$ & $\mathrm{~g}$ \\
Volume & 800 & $\mathrm{~cm}^{3}$ \\
\hline
\end{tabular}

To make a passive brace active, to networked ESAs can be attached to the brace in an agonistic-antagonistic fashion, similar to the Biceps and Triceps skeletal muscles. Hence, when one network shrinks, the other one relaxes to perform flexion, and vice versa for extension. Figure 6 shows a conceptual schematic of such a design. 




Figure 6. Conceptual schematic of bio-inspired arrangement of networked ESAs.

Another important parameter of an ESA is its maximum allowable elongation. This amount of elongation should be defined in two distinct levels: Passive and active. The maximum amount of passive elongation is defined as how much ESA can be stretched (by an external force) without passing its yield point. The maximum amount of active elongation can be determined by the maximum initial distance between the two coils of an ESA in such a way that the ESA is still capable of shrinking. By dividing the maximum elongation over the length of an ESA, the maximum passive or active elongation ratio can be determined. Due to the arrangement of ESAs inside a network, the passive and active elongation ratios of a single ESA would be the same as those of a network. Our previous experiments showed an active elongation ratio of $14 \%$ whereas the passive elongation ratio was found to be around $35 \%$.

\subsubsection{Optimization of Single ESA}

Before starting the optimization process, the categorical variables flux charge density has to be experimentally quantified. As mentioned before, to prepare the flexible permanent magnet, a mixture of PDMS and magnetic particles were cured inside a 3D printed mold while the whole mixture was exposed to a strong external magnetic field. However, ratio between magnetic particles and PDMS plays a very important interdisciplinary role, i.e., both in magnetic and mechanical domain. By increasing the ratio of magnetic particles, the flux charge density increases that would lead to enhancement of the magnetic field and the force generated by the coils. However, in mechanical domain, this increase in the magnetic particle ratio, affects the Young Modulus (i.e., elasticity) of the flexible magnet as well as its yield point, which in turn, affects the maximum passive elongation ratio of the permanent magnet.

Figure 7 shows components of a representative single ESA, its flexible magnetic core. We conducted mechanical tensile tests on several mixtures of PDMS and magnetic particles with different mixing ratios. The results suggested that mixing ratio of magnetic up to $28 \%$ (with respect to the weight compared to that of PDMS) would lead to safe (i.e., passive elongation ratio $>35 \%$ ). 


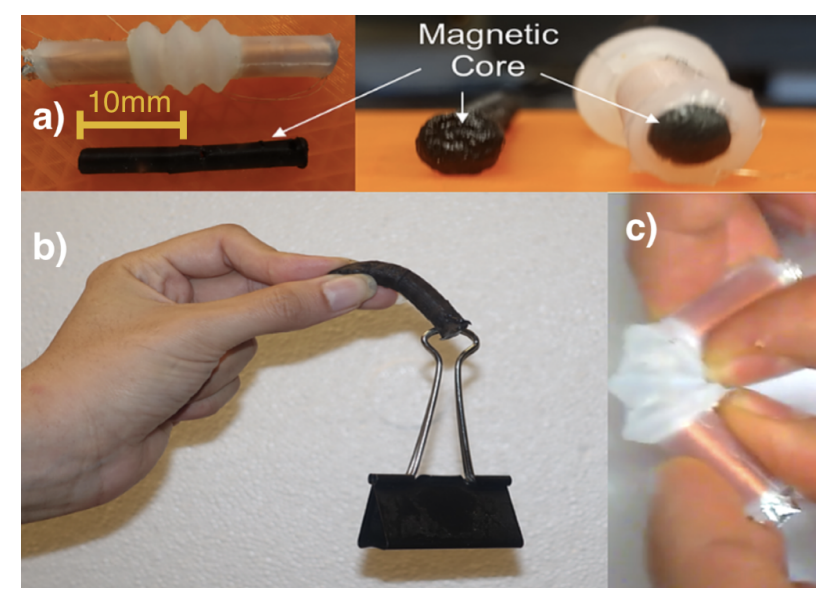

Figure 7. (a) A single ESA and the flexible magnet, (b) a flexible magnet lifting a metallic object and (c) a single ESA that is bent.

We prepared five flexible permanent magnet samples each with different mixing ration (between $8 \%$ to $28 \%$ with incremental mixing percentage of $4 \%$ of magnetic particles). For each sample, the flux charge density was measured by Magnetic Field Instrument (MFI), a device used to measure the magnetic field or flux around permanent magnets, coils, and electrical devices. The flux density for each mixing ratio was presented in an interval domain as the mixing ratio could not be precisely adjusted.

\subsubsection{Optimal Design of a Networked ESAs}

Once a single ESA has been optimally designed, we proceed with a network of ESAs by considering the performance parameters mentioned in Table 1.

The results on the optimal cross-section profile showed that although for a stacked network of ESAs, the triangular cross-section profile makes the largest magnetic field and force at the middle of solenoid compared to the other profiles, it also consumes the most electrical power such that after normalizing and obtaining the ratio, the triangular profile is the least efficient section profile while the circular one is the most.

We also considered the effect of packing density of the profiles since all of the coil's section profiles do not cover the available space entirely and there are some free spaces among the profiles for circular, octagonal and pentagonal arrangements. For this purpose the packing density coefficient $(\lambda)$ was defined and calculated for each section profiles. After applying the packing density coefficient to the previous findings we found that the hexagonal arrangement for the coil's section profile is the most efficient one due to have the largest amount $(\lambda=1.156)$ among all section profiles.

\section{Results}

Regarding the optimization of the soft permanent magnet as mentioned earlier we prepared five flexible permanent magnet samples each with different mixing ratios (between $8 \%$ to $28 \%$ with incremental mixing percentage of $4 \%$ of magnetic particles). For each sample, the flux charge density was measured by Magnetic Field Instrument (MFI), a device used to evaluate the magnetic field or flux produced around permanent magnets, solenoids, and electrical devices. The flux density for each mixing ratio was presented in an interval domain as the mixing ratio could not be precisely adjusted. The result is shown in Table 2. 
Table 2. Flux density and maximum passive elongation ratio of flexible PDMS-based permanent magnet with different magnetic particles ratio.

\begin{tabular}{ccc}
\hline Mixing Ratio (\%) & Flux Density (Tesla) & Maximum Passive Elongation Ratio (\%) \\
\hline $8-12$ & $0.4-0.5$ & $70-73$ \\
$12-16$ & $0.5-0.57$ & $60-70$ \\
$16-20$ & $0.57-0.63$ & $48-60$ \\
$20-24$ & $0.63-0.83$ & $40-48$ \\
$24-28$ & $0.83-0.9$ & $35-40$ \\
\hline
\end{tabular}

Result of the optimization problem for a single ESA has been presented in Table 3.

Table 3. Optimal design characteristics of a single ESA.

\begin{tabular}{ccc}
\hline Characteristics & Value & Unit \\
\hline Inner diameter of flexible wire & 4 & $\mathrm{~mm}$ \\
Length of the coil & 10 & $\mathrm{~mm}$ \\
Cross-section profile & 400 & $\mathrm{~mm}^{2}$ \\
Coil volume & 500 & $\mathrm{~mm}^{3}$ \\
Mixing ratio of flexible magnet & $20-24$ & $\%$ \\
\hline
\end{tabular}

Concerning the optimization of the spatial network of actuators as illustrated in the previous section, the comparison is done among the magnetic field of the actuator's coil with various cross-section profiles (triangle, square, pentagon, hexagon, octagon, and circle) and the results is presented in Table 4.

Table 4. Comparison of the produced magnetic fields and the forces among various coil cross-section profiles.

\begin{tabular}{ccc}
\hline Coil Section Profile & Magnetic Field Out of One Loop Coil & Ratio of Magnetic Fields \\
\hline Triangle & $B_{\text {tri }}=\frac{3 \mu_{0} I}{2 \pi a_{\text {tri }}} \cos \theta_{\text {tri }}$ & $\frac{B_{\text {tri }}}{B_{\text {tri }}}=1$ \\
\hline Square & $B_{s q u}=\frac{4 \mu_{0} I}{2 \pi a_{\text {squ }}} \cos \theta_{\text {squ }}$ & $\frac{B_{\text {squ }}}{B_{\text {tri }}}=0.955$ \\
\hline Pentagon & $B_{\text {penta }}=\frac{5 \mu_{0} I}{2 \pi a_{\text {penta }}} \cos \theta_{\text {penta }}$ & $\frac{B_{\text {penta }}}{B_{\text {tri }}}=0.946$ \\
\hline Hexagon & $B_{\text {hexa }}=\frac{6 \mu_{0} I}{2 \pi a_{\text {hexa }}} \cos \theta_{\text {hexa }}$ & $\frac{B_{\text {hexa }}}{B_{\text {tri }}}=0.943$ \\
\hline Octagon & $B_{\text {octa }}=\frac{8 \mu_{0} I}{2 \pi a_{\text {octa }}} \cos \theta_{\text {octa }}$ & $\frac{B_{\text {penta }}}{B_{\text {tri }}}=0.940$ \\
\hline Circle & $B_{\text {cir }}=\frac{\mu_{0} I}{2 R}$ & $\frac{B_{\text {cir }}}{B_{\text {tri }}}=0.939$ \\
\hline
\end{tabular}

To compare the efficiency of the profiles, the power consumption ratio needs to be taken into account. Considering same electric current, the only parameter affects the power consumption would the wire length. For the same cross section areas the perimeter ratio of various section profiles are listed in Table 4. Therefore, the calculated force ratios need to be normalized with perimeter ratios. Table 5 compares the produced force over perimeter ratio $\left(\frac{F}{P}\right)$ for aforementioned profiles. Another factor contributes to the final output of the network is packing density. Packing density is defined as the ratio of the cross-section taken up by the coils' wire section profiles to the available space. The maximum amount of packing density is for triangular, square, and hexagonal configuration which is one, means that the available space is absolutely filled with the coils. The remaining section profiles, i.e., pentagonal, octagonal and circular ones do not completely cover the available space and have less than 1 packing density. Packing densities for all section profiles are listed in Table 5. 
Table 5. Comparison of the produced magnetic fields and the forces among various coil cross-section profiles.

\begin{tabular}{ccc}
\hline Coil Section Profile & Force over Perimeter Ratio $\left(\frac{F}{P}\right)$ & $\left(\frac{F}{P}\right) \times \lambda$ \\
\hline Triangle & $\frac{\left(\frac{F}{P}\right)_{t r i}}{\left(\frac{F}{P}\right)_{t r i}}=1$ & 1 \\
\hline Square & $\frac{\left(\frac{F}{P}\right)_{s q u}}{\left(\frac{F}{P}\right)_{t r i}}=1.089$ & 1.089 \\
\hline Pentagon & $\frac{\left(\frac{F}{P}\right)_{\text {penta }}}{\left(\frac{F}{P}\right)_{t r i}}=1.132$ & 0.977 \\
\hline Hexagon & $\frac{\left(\frac{F}{P}\right)_{h e x a}}{\left(\frac{F}{P}\right)_{t r i}}=1.152$ & 1.152 \\
\hline Octagon & $\frac{\left(\frac{F}{P}\right)_{c t a}}{\left(\frac{F}{P}\right)_{t r i}}=1.178$ & 0.960 \\
\hline Circle & $\frac{\left(\frac{F}{P}\right)_{c i r}}{\left(\frac{F}{P}\right)_{t r i}}=1.208$ & 1.096 \\
\hline
\end{tabular}

Comparing the right column of Table 5 indicates that the hexagonal section profile is the most efficient one in terms of magnetic field and force output considering the power consumption and circular profile has the second rank.

\section{Discussion}

In this paper, the optimal design for a network of novel ESA was presented. The novel electromagnetic soft actuator operates based on the working principle of solenoids, that consists of two antagonistically located coils made of flexible wires and share a flexible permanent magnetic core.

It was shown that by reducing the size of these ESAs, the force to volume size ratio increases, which suggest a network of miniaturized ESAs would achieve higher amount of output force compare to a single ESA with the same size of the whole network. In this work this was numerically tested for a case study of an active elbow brace.

The goal was to achieve optimal design of a single ESA and consequently optimal design of a networked ESAs to achieve the maximum output torque.

The result showed that having a network of ESAs as drive train for an active brace, we can satisfy the performance parameters, for supporting the elbow joint of a patient with decreased muscle performance and mobility.

This suggests that with the available manufacturing process discussed in this paper, the actuation technology based on electromagnetic soft actuators can be used as drive trains in robotic prosthesis and robotic exoskeletons, to support patients with decreased muscle function at their affected joints. Our future endeavors are focused in enhancing further the produced torque by the ESAs, in order to be utilized for robotic prosthetics and exoskeletons in patients with complete loss of muscle function.

This actuation technology is uniquely suitable in rehabilitation and/or force augmentation applications for those mobility impaired patients that have not completely lost the ability to move their affected joints and would need some extra help to recover or be able to perform their daily tasks. Considering the huge population of these types of mobility impaired patients (e.g., stroke patients, peripheral arterial disease, traumatic injuries, neuropathies, senescence and frailty) electromagnetic soft actuators provide novel potential solutions, for wearable and next-to-skin type of assistive technologies, at low production cost, safe, portable and yet sufficiently powerful with low power requirement and high bandwidth.

As another case study, and considering the unique scale ability of the proposed actuation method, we will also consider a prosthetic finger, where the network of soft actuators can be linked to a tendon mechanism to bi-directionally move a finger. The return motion can then be done through a pre-loaded spring. 
Planning for the future, we will manufacture the active brace powered with the network of ESAs and test its potentials in rehabilitation trials, such as those for elbow stiffness or iso-kinetic motion for elbow spasticity and other motor dysfunctions from various medical pathologies.

Author Contributions: Conceptualization, A.J.; methodology, A.J.; software, N.E.; validation, A.J. and N.E.; formal analysis, N.E.; investigation, A.J.; resources, A.J.; data curation, N.E.; writing-original draft preparation, N.E.; writing — review and editing, A.J.; visualization, A.J.; supervision, A.J.; project administration, A.J.; funding acquisition, A.J. All authors have read and agreed to the published version of the manuscript.

Funding: This work was supported in part by the National Science Foundation NSF under Grant number 1840834.

Conflicts of Interest: The authors declare no conflict of interest.

\section{Abbreviations}

The following abbreviation is used in this manuscript:

ESA Electromagnetic Soft Actuator

\section{References}

1. Haddadin, S.; Albu-Schäffer, A.; Hirzinger, G. Safety Evaluation of Physical Human-Robot Interaction via Crash-Testing. Robot. Sci. Syst. 2007, 3, 217-224.

2. De Santis, A.; Siciliano, B.; De Luca, A.; Bicchi, A. An atlas of physical human-robot interaction. Mech. Mach. Theory 2008, 43, 253-270. [CrossRef]

3. Haddadin, S.; Albu-Schäffer, A.; Hirzinger, G. Requirements for safe robots: Measurements, analysis and new insights. Int. J. Robot. Res. 2009, 28, 1507-1527. [CrossRef]

4. Alami, R.; Albu-Schäffer, A.; Bicchi, A.; Bischoff, R.; Chatila, R.; De Luca, A.; De Santis, A.; Giralt, G.; Guiochet, J.; Hirzinger, G.; et al. Safe and dependable physical human-robot interaction in anthropic domains: State of the art and challenges. In Proceedings of the 2006 IEEE/RSJ International Conference on Intelligent Robots and Systems, Beijing, China, 9-13 October 2006; pp. 1-16.

5. Suzumori, K.; Endo, S.; Kanda, T.; Kato, N.; Suzuki, H. A bending pneumatic rubber actuator realizing soft-bodied manta swimming robot. In Proceedings of the 2007 IEEE International Conference on Robotics and Automation, Rome, Italy, 14 April 2007; pp. 4975-4980.

6. Polygerinos, P.; Lyne, S.; Wang, Z.; Nicolini, L.F.; Mosadegh, B.; Whitesides, G.M.; Walsh, C.J. Towards a soft pneumatic glove for hand rehabilitation. In Proceedings of the 2013 IEEE/RSJ International Conference on Intelligent Robots and Systems, Tokyo, Japan, 7 November 2013; pp. 1512-1517.

7. Yap, H.K.; Lim, J.H.; Nasrallah, F.; Goh, J.C.; Yeow, R.C. A soft exoskeleton for hand assistive and rehabilitation application using pneumatic actuators with variable stiffness. In Proceedings of the 2015 IEEE International Conference on Robotics and Automation (ICRA), Seattle, WA, USA, 30 May 2015; pp. 4967-4972.

8. Mata, A.; Fleischman, A.J.; Roy, S. Characterization of polydimethylsiloxane (PDMS) properties for biomedical micro/nanosystems. Biomed. Microdevices 2005, 7, 281-293. [CrossRef] [PubMed]

9. Rodrigue, H.; Wang, W.; Kim, D.R.; Ahn, S.H. Curved shape memory alloy-based soft actuators and application to soft gripper. Compos. Struct. 2017, 176, 398-406. [CrossRef]

10. Fang, L.; Fang, T.; Liu, X.; Chen, S.; Lu, C.; Xu, Z. Near-Infrared Light Triggered Soft Actuators in Aqueous Media Prepared from Shape-Memory Polymer Composites. Macromol. Mater. Eng. 2016, 301, 1111-1120. [CrossRef]

11. Choi, H.; Ryew, S.; Jung, K.; Kim, H.; Jeon, J.W.; Nam, J.; Maeda, R.; Tanie, K. Microrobot actuated by soft actuators based on dielectric elastomer. In Proceedings of the IEEE/RSJ International Conference on Intelligent Robots and Systems, Lausanne, Switzerland, 4 October 2002; Volume 2; pp. 1730-1735.

12. Gu, G.Y.; Gupta, U.; Zhu, J.; Zhu, L.M.; Zhu, X. Modeling of viscoelastic electromechanical behavior in a soft dielectric elastomer actuator. IEEE Trans. Robot. 2017, 33, 1263-1271. [CrossRef]

13. Nguyen, C.T.; Phung, H.; Nguyen, T.D.; Jung, H.; Choi, H.R. Multiple-degrees-of-freedom dielectric elastomer actuators for soft printable hexapod robot. Sensors Actuators A Phys. 2017, 267, 505-516. [CrossRef] 
14. Miriyev, A.; Stack, K.; Lipson, H. Soft material for soft actuators. Nat. Commun. 2017, 8, 1-8. [CrossRef] [PubMed]

15. Lipson, H. Challenges and opportunities for design, simulation, and fabrication of soft robots. Soft Robot. 2014, 1, 21-27. [CrossRef]

16. Do, T.N.; Phan, H.; Nguyen, T.Q.; Visell, Y. Miniature soft electromagnetic actuators for robotic applications. Adv. Funct. Mater. 2018, 28, 1800244. [CrossRef]

17. Yang, Y.P.; Liu, J.J.; Ye, D.H.; Chen, Y.R.; Lu, P.H. Multiobjective optimal design and soft landing control of an electromagnetic valve actuator for a camless engine. IEEE/ASME Trans. Mech. 2012, 18, 963-972. [CrossRef]

18. Ebrahimi, N.; Nugroho, S.; Taha, A.F.; Gatsis, N.; Gao, W.; Jafari, A. Dynamic actuator selection and robust state-feedback control of networked soft actuators. In Proceedings of the 2018 IEEE International Conference on Robotics and Automation (ICRA), Brisbane, Australia, 25 May 2018; pp. 2857-2864.

19. Jafari, A.; Ebrahimi, N. Electromagnetic Soft Actuators. U.S. Patent App. 16/457,452, 2 January 2020.

20. Ebrahimi, N.; Schimpf, P.; Jafari, A. Design optimization of a solenoid-based electromagnetic soft actuator with permanent magnet core. Sens. Actuators A Phys. 2018, 284, 276-285. [CrossRef]

21. Liu, D.K.C.; Friend, J.; Yeo, L. A brief review of actuation at the micro-scale using electrostatics, electromagnetics and piezoelectric ultrasonics. Acoust. Sci. Technol. 2010, 31, 115-123. [CrossRef]

22. Azzerboni, B.; Asti, G.; Pareti, L.; Ghidini, M. Magnetic Nanostructures in Modern Technology, Chapter 2-Spintronics, Magnetic MEMS and Recording; Springer: Dordrecht, The Netherlands, 2007; pp. 237-306.

23. Kemp, B.; Grzegorczyk, T.; Kong, J. Lorentz force on dielectric and magnetic particles. J. Electromagn. Waves Appl. 2006, 20, 827-839. [CrossRef]

24. Pappas, P. The original Ampere force and Biot-Savart and Lorentz forces. Il Nuovo Cimento B (1971-1996) 1983, 76, 189-197. [CrossRef]

25. Bryce, C.D.; Armstrong, A.D. Anatomy and biomechanics of the elbow. Orthop. Clin. N. Am. 2008, 39, 141-154. [CrossRef] [PubMed]

(C) 2020 by the authors. Licensee MDPI, Basel, Switzerland. This article is an open access article distributed under the terms and conditions of the Creative Commons Attribution (CC BY) license (http:/ / creativecommons.org/licenses/by/4.0/). 